\title{
Interfacial Structure in Epitaxial Perovskite Oxides on (001) Ge Crystal
}

\author{
Xuan Shen, ${ }^{1,2}$ K. Ahmadi-Majlan, ${ }^{3}$ Joseph H. Ngai, ${ }^{3}$ Di Wu, ${ }^{2}$ and Dong Su${ }^{1}$ \\ ${ }^{1 .}$ Center for Functional Nanomaterials, Brookhaven National Laboratory, Upton, NY 11973, USA \\ 2. National Laboratory of Solid State Microstructures and Department of Materials Science and \\ Engineering, Nanjing University, Nanjing, 210093, China \\ 3. Department of Physics, University of Texas at Arlington, 502 Yates Street, Arlington, TX 76019, USA
}

Integration of high-quality epitaxial perovskite oxides on semiconductors have been achieved due to the recently development of Molecular Beam Epitaxy (MBE).[1] For the hetero-epitaxial thin film system, as the film grows up to a certain thickness, interfacial dislocations are expected to form to relax the strain.[2] Intensive studies, both experimentally and theoretically, have been reported that these interfacial dislocations are crucial for some physical properties, such as dielectricity, polarization, and magnetization. Previous work have been focused on perovskite-on-perovskite system, while the nature of interfacial dislocations in the perovskite-on-semiconductor system has never been reported.

In this work, we have applied aberration corrected transmission electron microscopy (TEM) and scanning TEM (STEM) techniques to investigate the interfacial structure of $\mathrm{SrZr}_{0.68} \mathrm{Ti}_{0.32} \mathrm{O}_{3}$ (SZTO) perovskite thin films on (001) Ge single crystal wafers, The atomic resolution STEM image and selected area electron diffraction (SAED) pattern indicated the epitaxial crystallographic relation of (001)[100 $]_{\mathrm{SZTO}} / /(001)[110]_{\mathrm{Ge}}$ with abrupt interface. The misfit dislocation with Burgers vector of $1 / 2 \boldsymbol{a}$ $[0 \overline{1} 1]_{\text {SzTO }}$ was then observed at the SZTO film close to the interface, coupled with a terrace step of Ge. Using the STEM-EELS technique, we found the Ti diffusion into substrate at the dislocation core [Fig. 1]. However, this $1 / 2 \boldsymbol{a}<011>$ type dislocation could be the projection of $<111>$ direction which will be determined from the perpendicular direction (plane-view) sample. In the plane-view characterization, threading dislocations were observed with Burgers vectors of types $\boldsymbol{a}<100>$ and $1 / 2 \boldsymbol{a}<110>$. Considering the misfit dislocation with a Burgers vector of $1 / 2 \boldsymbol{a}$ [011], the $1 / 2 \boldsymbol{a}$ [011] misfit dislocation can be interpreted as the projection of the in-plane segment of $1 / 2 \boldsymbol{a}$ [1 $1 \overline{1} 1]$ dislocation at the (100) plane. We also found the dislocation reaction from two partial dislocations to one perfect dislocation $1 / 2 \boldsymbol{a}[1 \overline{1} 1]+1 / 2 \boldsymbol{a}$ $[11 \overline{1}]=\boldsymbol{a}$ [100], which leads to the formation of threading dislocation with Burgers vector of $\boldsymbol{a}$ [100] [Fig. 2]. In addition, we found the coupling of dislocation half-loop with anti-phase boundary (APB, shift vector of $1 / 2 \boldsymbol{a}<111>$ ) induced by lattice terrace of $\mathrm{Ge}$ and they can decouple after annealing. The possible models based on the half-loop theory are proposed for the dislocation reaction and the coupling behavior.

We have used combined (S)TEM and modeling analysis to solve the nature of the interfacial dislocation in perovskite-on-semiconductor system, which gives insight into the accommodation of the misfit strain from the view of TEM [3].

\section{References}

[1] R. A. McKee, F. J. Walker, and M. F. Chisholm, Science 293, 468 (2001).

[2] J. W. Matthews, and A. E. Blakeslee, J. Cryst. Growth 27, 118 (1974).

[3] X. Shen et al., Appl. Phys. Lett. 106, 032903 (2015).

[4] Electron microscopy studies were performed at Center for Functional Nanomaterials, Brookhaven National Laboratory supported by the U.S. Department of Energy, Office of Basic Energy Sciences, under contract no. DEAC02-98CH10886. X. S. is grateful for the financial support of the China Scholarship Council and Brookhaven National Laboratory for his exchange program. K. A. -M. and J. H. 
N. acknowledge the support of The University of Texas at Arlington. D. W. thanks National Key Basic Research Program of China (2015CB921203) for support. The authors thank Dr. Woodhead for the proof reading.
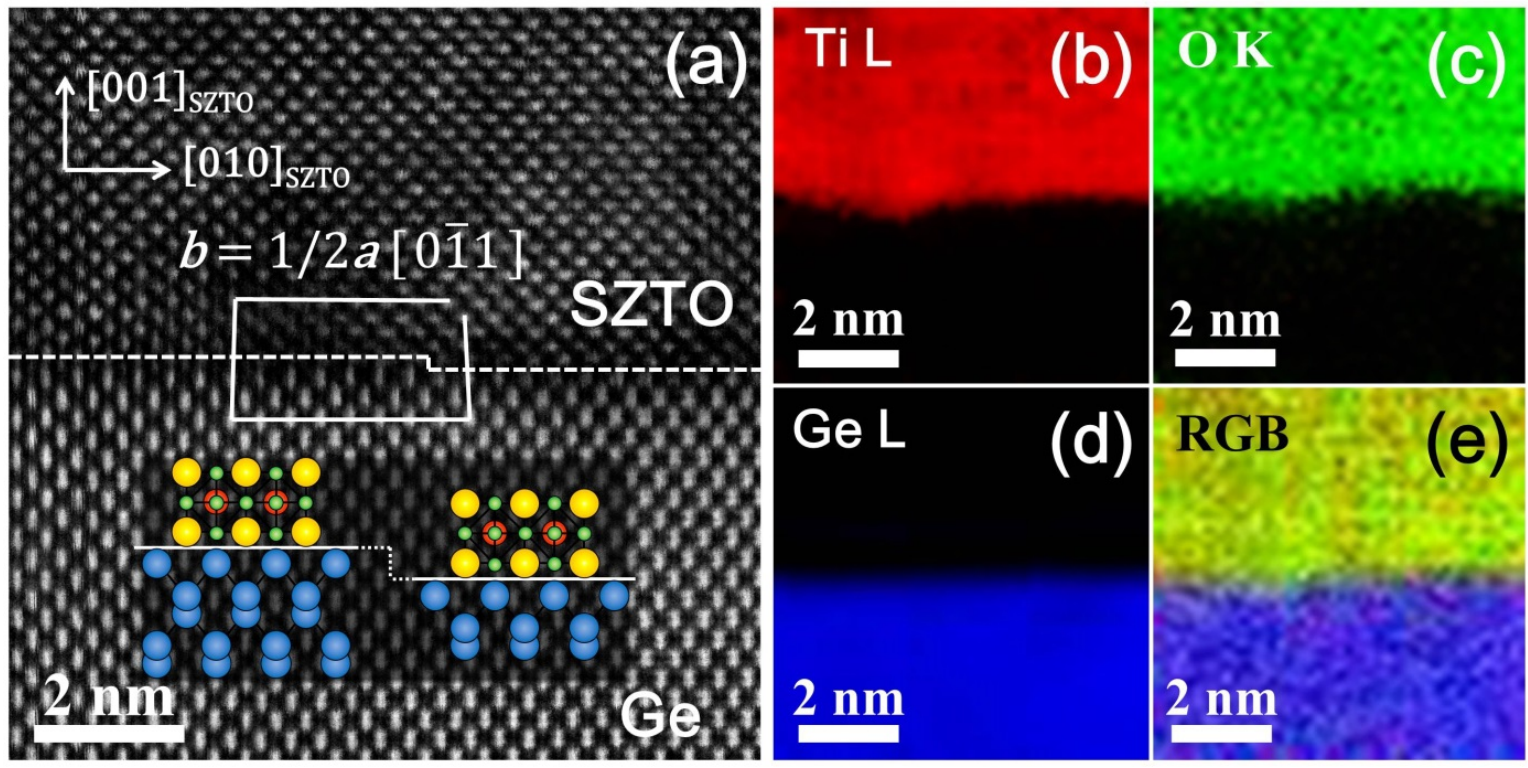

Figure 1. (a) Cross-sectional STEM image with Burgers vector along [011] direction. The Burgers circuit is drawn around the dislocation core. Schematic diagram of step and terrace structure is shown in the inset. Elemental maps (50 pixels by 50 pixels) from STEM EELS of Ti (red), O (green), Ge (blue) and their RGB mixture are shown in (b), (c), (d), and (e), respectively.
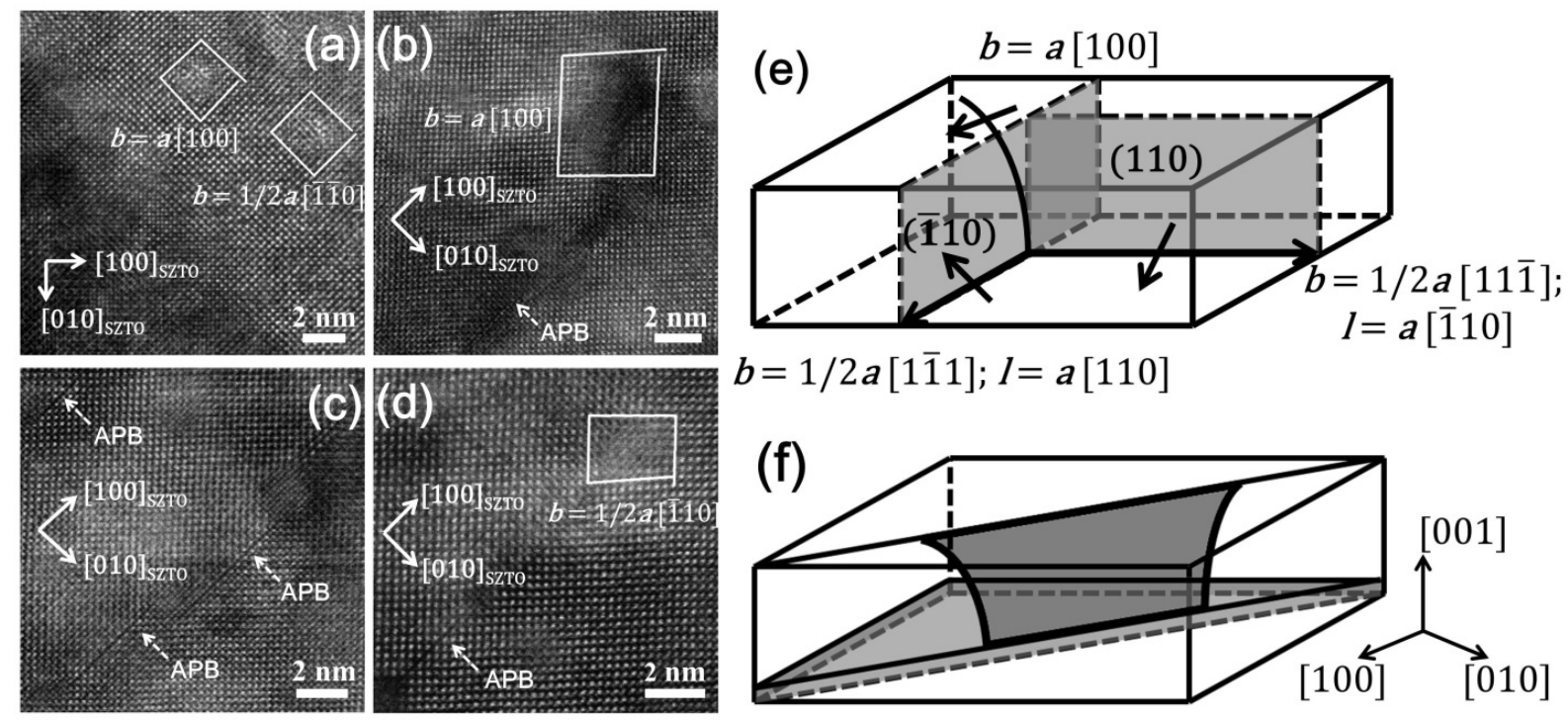

$b=1 / 2 a[1 \overline{1} 1] ; l=a[110]$

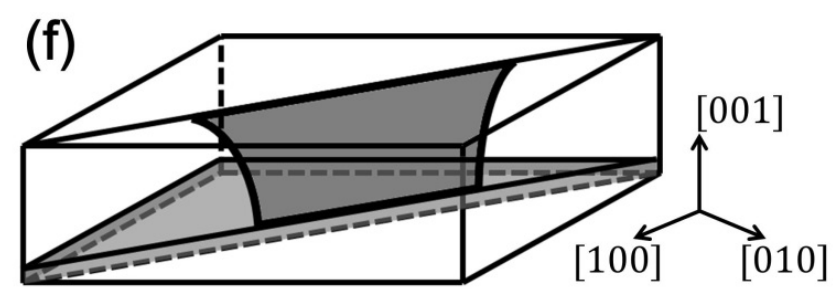

Figure 2. (a) Plane-view HRTEM image of SZTO thin films. HR-STEM images of the plane view are shown in (b), (c), and (d). The APBs are indicated by white dashed arrows. Schematic diagrams of dislocation dissociation and coupling behavior between APBs and dislocations are shown in (e) and (f), respectively. 